\title{
On wavelet techniques in atmospheric sciences
}

\author{
Margarete Oliveira Domingues
}

\author{
LAC/CTE \\ National Institute of Space Research - INPE \\ 12201-970 São José dos Campos, São Paulo, Brazil
}

Odim Mendes Jr.

$D G E / C E A$

National Institute of Space Research - INPE

12201-970 São José dos Campos, São Paulo, Brazil

Aracy Mendes da Costa

$D G E / C E A$

National Institute of Space Research - INPE

12201-970 São José dos Campos, São Paulo, Brazil

\begin{abstract}
Wavelet analysis has been formalized extensively due to the efforts of mathematicians, physics and engineers in the last two decades. It has generated a tremendous interest in these communities both in theoretical and applied areas, in such a way that wavelet analysis is also considered now as a nucleus of shared aspirations and ideas. Initially applied to seismic signal studies in geophysics in the 80 's, wavelet techniques have been explored in the atmospheric sciences since the pioneer applications in turbulence studies. If one decides to apply the wavelet analysis to a given signal, it is worthwhile to assess the actual need of the technique itself and the best way to perform it. In atmospheric signal applications, two main directions have been followed: the singularity and the variance analysis. In this paper the potential uses of this tool supported by some recently published works in the field of atmospheric sciences are discussed. Therefore, initially the characteristics and main properties of the wavelet analysis are presented, focusing on those that are mostly used in the analysis of atmospheric signals. Continuous and discrete wavelet transforms are also discussed, as well as the scalograms and the variance analysis. Finally some examples of wavelet analysis applied to a wide range of atmospheric science phenomena are presented.
\end{abstract}




\section{Introduction}

The wavelet analysis is a mathematical technique which is very useful for numerical analysis and manipulation of multidimensional discrete signal sets. Originally applied in geophysics to the analysis of seismic signals, the wavelet transforms were better and broadly formalized thanks to mathematicians, physicists, and engineers efforts (Morlet, 1983). Therefore, the use of wavelet techniques in data analysis has exponentially grown, since it represents a synthesis of old techniques associated with robust mathematic results and efficient computational algorithms under the interest of a broad community (Daubechies et al., 1992). In a rapidly developing field, overview papers are particularly useful, and several good ones concerning to wavelets are already available (Daubechies, 1992; Chui, 1992a,b; Jawerth and Sweldens, 1994; Chui, 1994; Strang and Nguyen, 1996).

In atmospheric applications, the main characteristic of the wavelet technique is the introduction of the time-frequency decomposition. A well known example of such a behavior can be found in the musical structure, where it has been interpreted as events localized in time. Although it belongs to a more complex structure, a piece of music can be understood as a set of musical notes characterized by four parameters: frequency, time of occurrence, duration and intensity (Daubechies, 1992; Lau and Weng, 1995).

In the last decades wavelet technique has been extensively adopted in the atmospheric sciences. Among the pioneer applications stand out the turbulence studies (Meneveau, 1991; Farge, 1992; Gao and Li, 1993; Katul et al., 1994) and the interannual and inter-seasonal variations of the South Oscillations and El Niño (Gambis, 1992).

When wavelet analysis is used to study a given signal, it is essential to choose the best wavelet representation for the signal under study. There are many relevant texts on this subject, for example Foufola-Georgoiu and Kumar (1994); Sweldens and Schröder (1995); Lau and Weng (1995); Strang and Nguyen (1996) and Torrence and Compo (1998). In statistical applications, the wavelet analysis has also been deeply studied (Houdré, 1994; Percival and Walden, 2000; Vidakovic, 2000). In the Appendix some web pages containing useful information and software about wavelet technique are presented.

In this context, two main directions have been followed for the atmospheric signal analysis: the singularity analysis and the variance wavelet analysis or, in analogy with Fourier terminology, the wavelet spectrum.

The purpose of this paper is to help the potential users of this tool offering a tutorial based on some recently published articles in the field. In the following sessions some basic concepts on wavelet analysis, scalogram and wavelet variance are present, as well as some hints to properly select the wavelet family. 


\section{Wavelet concepts}

The word wavelet was adopted to express the idea of "small waves" in an intuitive meaning associated with localized waves, i.e., , wave-like functions whose values increase and decrease in a short period of the domain. In order to a function be called a wavelet it must satisfy the following conditions.

1) The integral of the wavelet function, usually denoted by $\psi$, must be zero

$$
\int_{-\infty}^{\infty} \psi(t) d t=0
$$

This assures that the wavelet function has a wave shape and it is known as the admissibility condition.

2) The wavelet function must have unitary energy, i.e.,

$$
\int_{-\infty}^{\infty}|\psi(t)|^{2} d t=1
$$

This assures that the wavelet function has compact support or has a fast amplitude decay (in a physical vocabulary $e$-folding time), warranting a physical domain localization.

The Fourier transform is a widely used tool for stationary signal analysis where all frequencies have an infinite coherence in time. The Fourier analysis brings only global information which is not sufficient to detect compact patterns. Gabor introduced a local Fourier analysis, in which a sliding window, leads to a time frequencyanalysis. This method is only applicable to situations where the coherence time is independent of the frequency. This is the case, for instance, for "singing-signals" which have their coherence time determined by the geometry of the oral cavity (Gabor, 1946). Morlet introduced the wavelet transform in order to have a coherence time proportional to the period (Meyer, 1989).

The wavelet transform is linear and covariant under translation and dilatation transform. The wavelet transform can be used in the analysis of non-stationary signals to obtain information on the frequency or scale variations of those signals and to detect its structures localization in time and/or in space. The time/space localization occurs because the wavelet function is defined in a finite interval. In this way, as the scale decreases, the wavelet functions of those scales are localized in shorter and shorter intervals. In each scale level all wavelet functions have the same form, only changing its localization points, i.e., they undergo a translation motion. Due to the double localization property of the wavelet function, the wavelet transform is said to be of local type in time-frequency, with time and frequency resolutions inversely proportional. Such behavior is shown in the graph of time/space $(x)$ vs frequency $(\xi)$ plane presented in Fig. 1. Proportional variations of time/space and frequency intervals are presented on the left for three different types of scale. On the right, 
the wavelet function is shown, when the scale parameter $j=0$, a dilatation for the mother-wavelet, when $j>0$ and a contraction of the mother-wavelet when $j<0$. Thus, the wavelet transform has this double localization property: in frequency and in time/space, with a compromise between them.

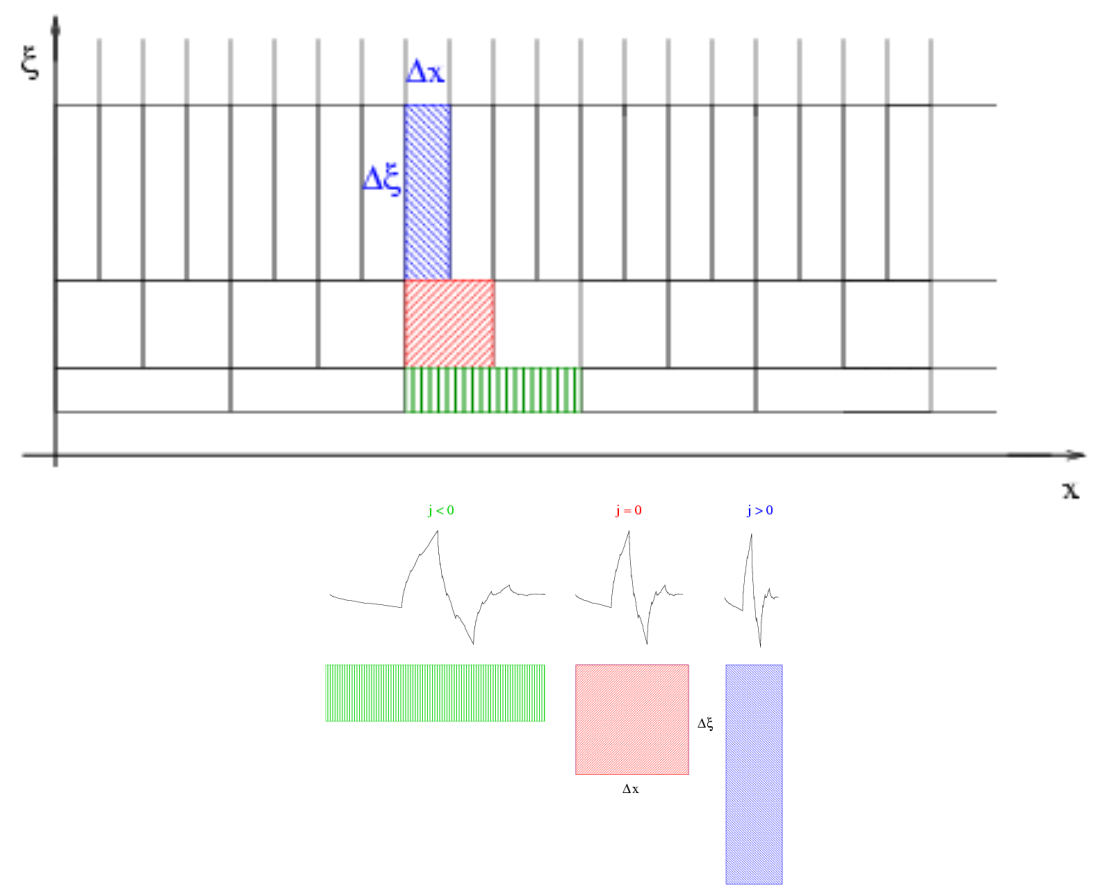

Figure 1. Time/space vs frequency plane representation. Proportional variations of time and frequency intervals are presented on the left. On the right, the dilatation/contraction of a wavelet function in those respective intervals is shown.

\section{Continuous wavelet transform (CWT)}

The CWT of a time series $f$ is defined by the integral transform,

$$
\mathfrak{W}_{f}^{\psi}(a, b)=\int_{-\infty}^{\infty} f(u) \bar{\psi}_{a, b}(u) d u \quad a>0,
$$

where

$$
\psi_{a, b}(u)=\frac{1}{\sqrt{a}} \psi\left(\frac{u-b}{a}\right)
$$

represents a chosen wavelet function family, named mother-wavelet. The parameter $a$ refers to a scale, $b$ is a translation parameter or localization of the motherwavelet function and $\bar{\psi}_{a, b}(u)$ is the conjugate complex of $\psi_{a, b}(u)$. The variation of $a$ has a dilatation effect (when $a>1$ ) and a contraction effect (when $a<1$ ) of the mother-wavelet function. Therefore, it is possible to analyze the long and short period features of the signal or the low and high frequency aspects of the signal. As $b$ varies, the function $f$ is locally analyzed in the vicinities of this point. 
Such a transform is called the continuous wavelet transform (CWT), because the scale and localization parameters assume continuous values. The CWT of a time series can be visually represented by an image or a field of isolines. It is also possible to get the inverse function of this transform, namely:

$$
\mathfrak{I W}_{f}^{\psi}(u)=\frac{1}{C_{\psi}} \int_{-\infty}^{\infty} \int_{0}^{\infty} \frac{1}{a^{2}} \mathfrak{W}_{f}^{\psi}(a, b) \bar{\psi}_{a, b}(u) d a d b
$$

where $C_{\psi}$ is a constant that depends on the chosen wavelet function.

The CWT is equivalent to a mathematician microscope, whose magnification is given by the inverse of the dilatation parameter and the optical ability is given by the choice of the mother-wavelet function (Foufola-Georgoiu and Kumar, 1994).

A Morlet mother-wavelet is formed by a plane wave modulated by a gaussian function and it is given by

$$
\psi(t)=\pi^{-\frac{1}{4}}\left(e^{i \xi t}-e^{-\frac{\xi^{2}}{2}}\right) e^{-\frac{t^{2}}{2}},
$$

where $\xi$ is a non dimensional value. Ordinarily $\xi$ is assumed to be equal to 5 to make the highest and lowest values of $\psi$ be approximately equal to $1 / 2$, thus the admissibility condition is satisfied (Daubechies, 1992). A graph of this function is presented in Fig. 2, in the left, the real part, and on the right, the complex one. Since this wavelet is a complex function it is possible to analyze the phase and the modulus of the decomposed signal.
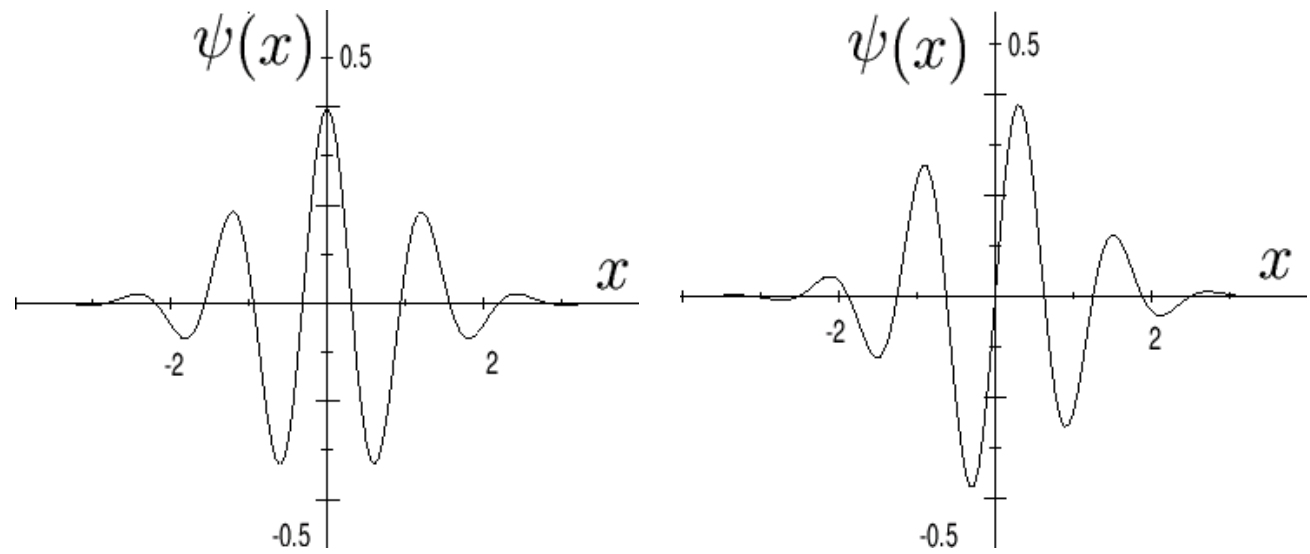

Figure 2. Graph of real and complex part of Morlet mother-wavelet

Another mother-wavelet known as mexican-hat or Maar is the second derivative of the gaussian probability density function, expressed as

$$
\psi(t)=\frac{2}{\sqrt{3} \pi^{\frac{1}{4}}} e^{-\frac{t^{2}}{2}}\left(1-t^{2}\right) .
$$


This function has an infinite support; nevertheless its effective support is in the interval $[-5,5]$ as can be seen in Fig. 3. It is a isotropic function in high-order dimension.

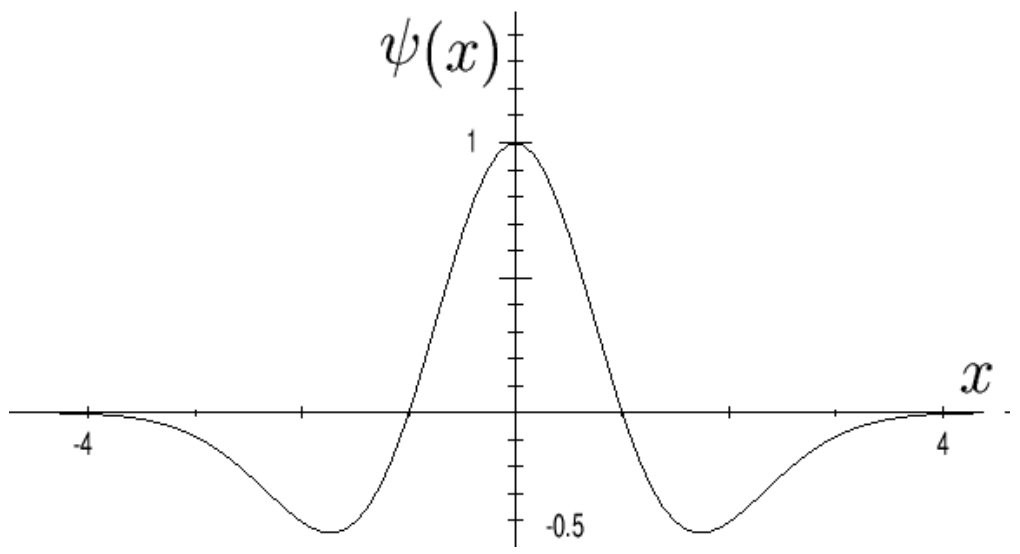

Figure 3. Graph of mexican-hat mother-wavelet .

\section{Discrete wavelet transform}

In the CWT derivation, most of the information related to close scales or times is redundant. These procedures result in a high computational cost, that in some cases, could be solved using an adequate discrete wavelet transform (DWT), which uses discrete values of scale $(j)$ and localization $(k)$. In such case, one may have redundant representations or not, depending on the discretization scheme used. To avoid redundancies, one can choose wavelet functions that form an orthogonal basis and define the DWT as

$$
\mathfrak{D}_{f_{k}^{j}}^{j}=\int_{-\infty}^{\infty} f(u) \bar{\psi}_{k}^{j}(u) d u
$$

where

$$
\psi_{k}^{j}(u)=2^{-\frac{j}{2}} \psi\left(2^{-j} u-k\right)
$$

Such sets of wavelet functions are orthogonal and their respective functions are translated and dilated.

Signals $f(u)$ are represented by series such as

$$
f(u)=\sum_{j=-\infty}^{\infty} \sum_{k=-\infty}^{\infty} d_{k}^{j} \psi_{k}^{j}(u),
$$

where $\psi_{k}^{j}(u)=\psi\left(2^{j} u-k\right)$ are wavelet functions and $d_{k}^{j}$ are wavelet coefficients

$$
d_{k}^{j}=\int f(u) \psi_{k}^{j}(u) d u
$$


As a property of the wavelet analysis, it is possible to show that the amplitude of the wavelet coefficients is associated with abrupt signal variations or "details" of higher frequency (Meyer, 1990; Daubechies, 1992; Chui, 1992b). On the other hand, the Fourier transform is unable to recognize this transition region. In the Fourier analysis only the presence of the involved frequencies is detected. No information on the spatial localization of these frequencies is given.

The most simple orthogonal mother-wavelet function is the Haar wavelet

$$
\psi(u)=\left\{\begin{array}{cl}
1, & 0 \leq u<\frac{1}{2} \\
-1, & 1 / 2 \leq u<1 \\
0, & \text { otherwise. }
\end{array}\right.
$$

The DWT using Haar wavelet detects signal abrupt variations, i.e., one localization feature in the physical space.

It is possible to build up wavelet functions using a mathematical tool known as multi-resolution analysis formed by a pair $\left\{V^{j}, \phi^{j}\right\}$, in such a way that there are sequences of embedded approximating spaces $V^{j} \subset V^{j-1}$ and the functions $\phi_{k}^{j}$ formed a Riesz basis for $V^{j}$ of $L^{2}(\mathbf{R} / \mathbf{Z})$ (Mallat, 1991; Daubechies, 1992; Jawerth and Sweldens, 1994), being

$$
V_{j}=\operatorname{span}\left\{\phi_{k}^{j}(x)\right\} .
$$

In this technique, a mother-wavelet function is generated from a scaling function. It obeys the scale relation

$$
\phi(x)=2 \sum_{k} h(k) \phi(2 x-k),
$$

where $\phi(x)$ is known as the scale function, and $h(k)$ is a low pass filter. Then, the mother-wavelet functions are build as

$$
\psi(x)=\sum_{k} g(k) \phi(2 x-k),
$$

where $g(k)=(-1)^{k+1} h(1-k)$ is a high-pass band filter. From this motherwavelet, it is possible to build up functions $\psi_{k}^{j}$ that can be dilated and contracted. They also formed a Riesz basis for the "detail" spaces $W^{j}=V^{j-1}-V^{j}$. This represents the difference of information between $V^{j}$ and $V^{j-1}$, i.e.,

$$
\left(\Pi^{j-1}-\Pi^{j}\right) f(x)=Q^{j} f(x)
$$

where the projections are

$$
\Pi^{j} f(x)=\sum_{k}\left\langle f, \phi_{k}^{j}\right\rangle \phi_{k}^{j}(x)
$$


and the details are

$$
Q^{j} f(x)=\sum_{k}\left\langle f, \psi_{k}^{j}\right\rangle \psi_{k}^{j}(x)
$$

This procedure allows that a basis with compact support and arbitrary smoothness degree can be build up. They form a orthogonal system, i.e.,

$$
\int_{-\infty}^{\infty} \psi\left(2^{-j} x-k\right) \psi\left(2^{-\ell} x-n\right) d x=\left\{\begin{array}{cl}
2^{-j} & \text { if } j=\ell \text { and } k=n \\
0 & \text { otherwise }
\end{array}\right.
$$

This means that no redundant informations are stored.

The Daubechies orthogonal wavelet functions are examples of that type of construction. Those functions do not have analytical expressions and are not symmetric. The $h(k)$ coefficients are zero for $k<0$ and for $k \geq 2 K$, where $K$ is related to the smoothness order of the wavelet. The support of $\phi=\phi_{K}$ is $\left[0, \frac{K}{2}-1\right]$. Table 1 presents this coefficients for $K=2,3$ and 4. Fig. 4 shows typical examples of Daubechies orthogonal function families for $K=3$ and 4 .

Table 1

Scale coefficient $h(k)$ for Daubechies orthogonal functions

\begin{tabular}{cc|c|c}
\hline \hline & $K=2$ & $K=3$ & $K=4$ \\
\hline $\mathrm{k}$ & $h(k)$ & $h(k)$ & $h(k)$ \\
\hline 0 & 0.341506350946110 & 0.235233603892082 & 0.162901714025649 \\
1 & 0.591506350946109 & 0.570558457915722 & 0.505472857545914 \\
2 & 0.158493649053890 & 0.325182500263116 & 0.446100069123380 \\
3 & -0.0915063509461096 & -0.0954672077841637 & -0.0197875131178224 \\
4 & & -0.0604161041551981 & -0.132253583684520 \\
5 & & 0.0249073356548795 & 0.0218081502370886 \\
6 & & & 0.0232518005354909 \\
7 & & & -0.00749349466518071 \\
\hline \hline
\end{tabular}

To obtain two dimensional multi-resolution analysis, the most commonly used method is the tensor product (Mallat, 1991; Daubechies, 1992). This procedure can be used to construct separable bi-orthogonal multi-resolution analysis of $L^{2}(\mathbf{T})$, where $T=\mathbf{R} / \mathbf{Z} \times \mathbf{R} / \mathbf{Z}$. Starting from an univariate orthogonal multi-resolution analysis $\left\{V^{j}, V^{j}\right\}$, the vector spaces

$$
\mathcal{V}^{j}=V^{j} \times V^{j}
$$



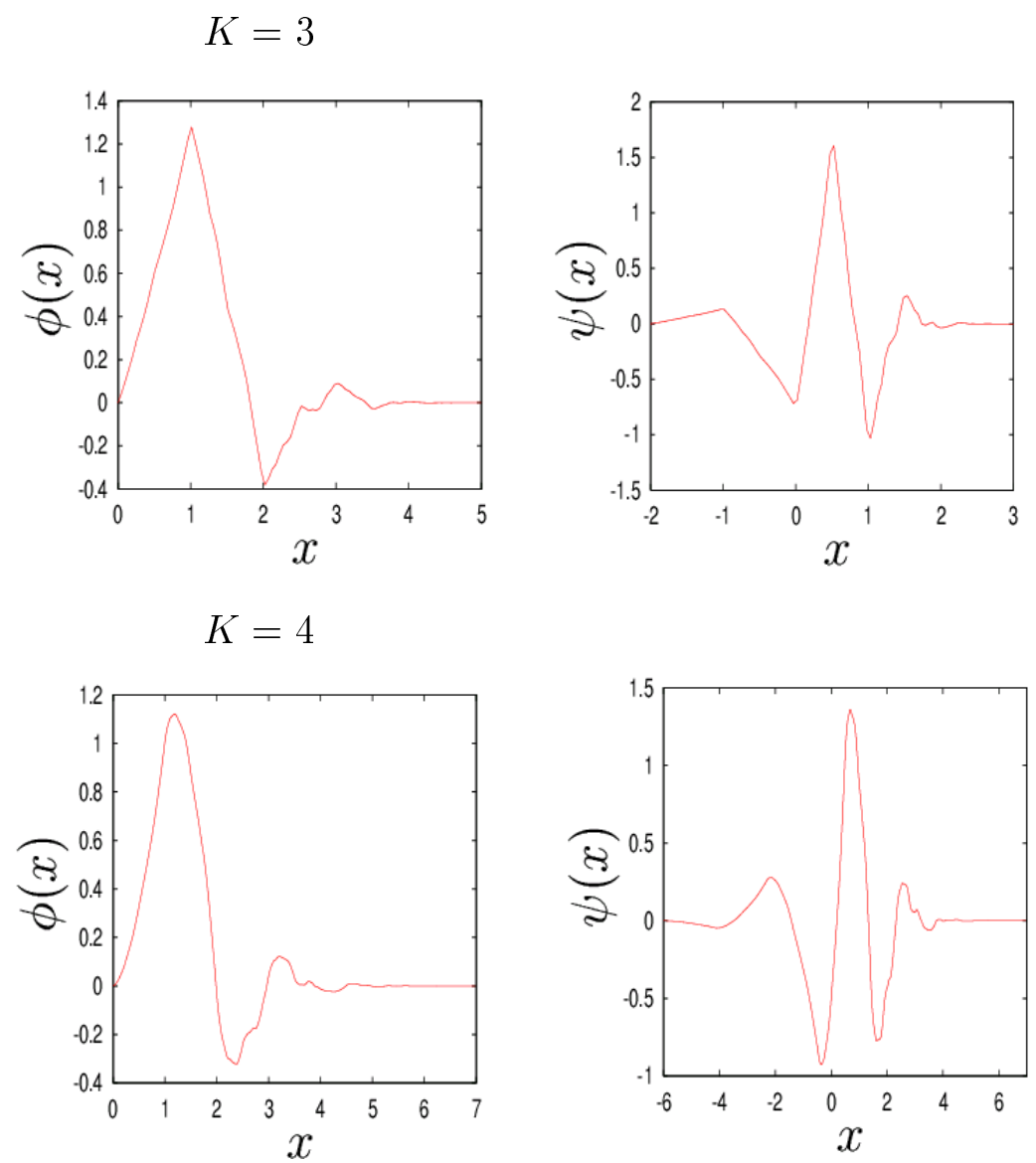

Figure 4. Typical examples of Daubechies orthogonal scale and wavelet function families. are formed by tensor product of two identical subspaces $V_{j}$, then

$$
\mathcal{V}^{j}=\operatorname{span}\left\{\Phi_{k, l}^{j}(x, y)=\phi_{k}^{j}(x) \phi_{\ell}^{j}(y)\right\}
$$

Consider also the vector spaces $\mathcal{W}^{j}$ spanned by the three families of wavelet $\Upsilon_{k, \ell}^{j}{ }^{(1)}(x, y)=\phi_{k}^{j}(x) \psi_{\ell}^{j}(y), \quad \Upsilon_{k, \ell}^{j}{ }^{(2)}(x, y)=\psi_{k}^{j}(x) \phi_{\ell}^{j}(y), \quad \Upsilon_{k, \ell}^{j}{ }^{(3)}(x, y)=\psi_{k}^{j}(x) \psi_{\ell}^{j}(y)$.

The following relations hold

$$
\mathcal{V}^{j} \subset \mathcal{V}^{j-1}, \quad \mathcal{V}^{j-1}=\mathcal{V}^{j} \oplus \mathcal{W}^{j}
$$

The difference of information between $\mathcal{V}^{j}$ and $\mathcal{V}^{j-1}$ is given by

$$
\left(\Pi^{j-1}-\Pi^{j}\right) f(x, y)=Q^{j(1)} f(x, y)+Q^{j^{(2)}} f(x, y)+Q^{j(3)} f(x, y),
$$

where the projections are

$$
\Pi^{j} f(x, y)=\sum_{k} \sum_{\ell}\left\langle f, \Phi_{k, \ell}^{j}\right\rangle \Phi_{k, \ell}^{j}(x, y)
$$


and three detail projections are

$$
Q^{j(a)} f(x, y)=\sum_{k} \sum_{\ell}\left\langle f, \Upsilon_{k, \ell}^{j}{ }^{(a)}\right\rangle \Upsilon_{k, \ell}^{j}{ }^{(a)}(x, y)
$$

for $a=1,2$ and 3 .

In order to get symmetry, it has been developed a procedure using two multiresolution analysis, formed by pairs $\left\{V^{j}, \phi^{j}\right\},\left\{V^{* j}, \phi^{* j}\right\}$, with defined orthogonal relations. This has led to the construction of the Daubechies bi-orthogonal spline families. This kind of wavelet family is usually used in numerical analysis or situations where symmetry is important. More details about these families see in Daubechies (1992).

\section{Scalogram and variance analysis}

The wavelet transform is a transform that preserves the energy. In analogy with the terminology adopted in Fourier analysis, the squared modulus of the wavelet coefficients of the CWT is called scalogram and the product of two CWT of distinct functions is called cross-scalogram (Flandrin, 1988). The scalogram informs if the analyzed signal has multi-scale characteristics and which scales participate in the processes depicted by the signal.

Focusing on the measurement and characterization of the local kinetic energy in each scale in turbulence flow, the variance wavelet analysis or the wavelet spectrum has been originally defined by Meneveau (1991) as

$$
S(a)=\int_{-\infty}^{\infty} \mathfrak{W}_{f}^{\psi}(a, t) d t
$$

It is also possible to derive the wavelet spectrum starting from the DWT . In such case, considering signals with zero average and $N=2^{J}$ elements, the total energy contained in each scale $j$ is expressed by

$$
E\left(k^{j}\right)=\frac{d s}{2 \pi \ln (2)} 2^{(j-J)} \sum_{k=1}^{2^{J-j}}\left[d_{k}^{j}\right]^{2},
$$

with the wave number

$$
k^{j}=\frac{2 \pi}{2^{j} d s},
$$

where $d s$ is the interval of the observed samples (Katul et al., 1994; Percival and Walden, 2000). In terms of the scale the resolution is performed only on octaves. In the Fourier analysis, the wave number is spaced linearly. The deviations of the energy around their mean value can be quantified by the variance of $E\left(k^{j}\right)$, which 
is essentially a fourth order moment (flatness) of the wavelet coefficient. Thus, the standard deviation of the energy is defined as

$$
\sigma_{E}\left(k^{j}\right)=\frac{d s}{2 \pi \ln (2) 2^{(J-j)+1}}\left\{\sum_{k=1}^{2^{J-j}}\left[d_{k}^{j}\right]^{4}-\sum_{k=1}^{2^{J-j}}\left[d_{k}^{j}\right]^{2}\right\}^{\frac{1}{2}} .
$$

In Fig. 5(a) a comparison between wavelet and Fourier spectra are presented for the zonal wind component from a micro-meteorological tower in Rebio Jaru (Amazon region). It is easily noticed that the wavelet spectrum is smoothed. Wavelet transform coefficients are influenced by local events; whereas in the Fourier transform, the coefficients are functions of the domain as a whole. This makes the wavelet spectrum a better measurement of the variance attributed to localized events. Another advantage of such spectrum is that it can be build up even when there are gaps in the time series. This makes the long period analysis easier and feasible. The local maximum in this spectrum provides information on scales in which important characteristics or coherent events contribute significantly.

(a) wavelet spectrum

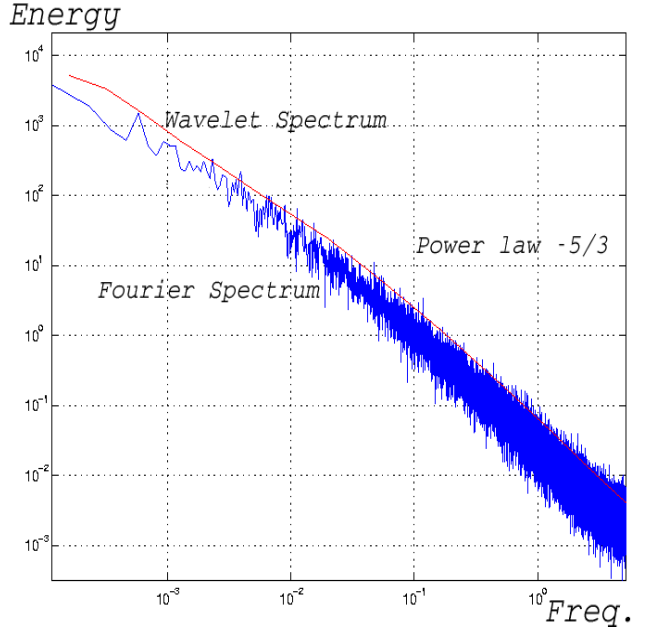

(b) wavelet co-spectrum

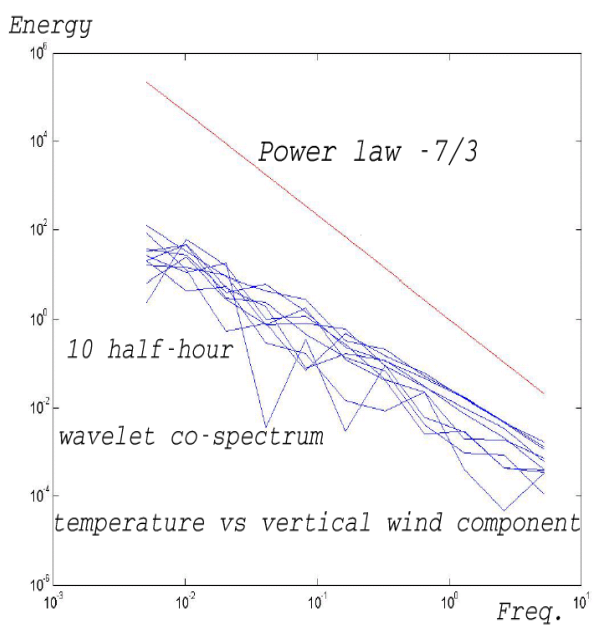

Figure 5. One example of waveletspectrum of the zonal wind component and co-spectrum of temperature and vertical wind component of turbulent signals from a micro-meteorological tower in Rebio Jaru (Amazon region) in (a) and (b) respectively. In (b), a standard power law function is also shown in the upper part.

Source: Domingues et al. (2002)

Similarly, it is also possible to define a wavelet co-spectrum of two functions $f$ and $g$ of their wavelet coefficients $d j$ in $j$ scales, by means of

$$
\mathcal{C}_{w}^{j}=\frac{d s}{2^{j} \ln (2)} \sum_{k=1}^{2^{j}} d_{k}^{j,(f)} d_{k}^{j,(g)},
$$

where $d_{k}^{j,(f)}$ and $d_{k}^{j,(g)}$ are the wavelet coefficients obtained in the wavelet transform 
of the functions $f$ an $g$ respectively.

In Fig. 5(b) wavelet co-spectra of temperature and vertical wind component for 30 minutes periods of part of one morning, from a micro-meteorological tower in Rebio Jaru (Amazon region), is presented. In the upper part of this graph a theoretical power law line for this kind of spectra data is also shown.

Some relations among wavelet coefficients and higher order moments, as symmetry and flatness, have also been defined (Meneveau, 1991; Katul et al., 1994; Percival and Walden, 2000; Vidakovic, 2000).

\section{How to choose a wavelet function?}

An issue that is always emerging in the application of wavelet techniques is the choice of the wavelet function appropriate to an specific signal. In order to demonstrate the possibilities of this transform and its dependence on the characteristics of the chosen wavelet, a synthetic sinusoidal signal which contains two different frequencies $(\pi / 8, \pi / 2)$ is analyzed by means of the mexican hat mother-wavelet and the Morlet mother-wavelet in Fig. 6.

In the upper panel of this figure the signal representation is given. In the others, the Fourier spectrum and after it the scalogram using Morlet mother-wavelet and mexican-hat mother-wavelet are presented. In Fourier spectrum only the frequency localization is given. No information about time localization is possible. In both wavelet scalograms, the time localization of the period changes can be clearly detected. It is also possible to observe that there is a better frequency localization of Morlet wavelet transform, although it has a worse time localization, this is expected by the Heisenberg's uncertainty principle (Daubechies, 1990). The wavelet variance can be used to determine the exact values of the characteristic frequencies. In the example this is possible only for the Morlet-wavelet, which has the best localization in frequency domain and could be compared with the frequencies showed in the Fourier spectrum. It is also possible to observe that the high values of the modulus of the wavelet coefficients indicate a transition region among different types of movements.

Some recommendations can be useful, although this procedure can be developed in several ways.

- The shape of the chosen wavelet function must translate the characteristics of the time series. For example, to represent a time series with abrupt variations or steps, the Haar wavelet may be the most convenient; in the analysis of time series with smoother variations, the mexican hat and Morlet wavelet can be recommended. 

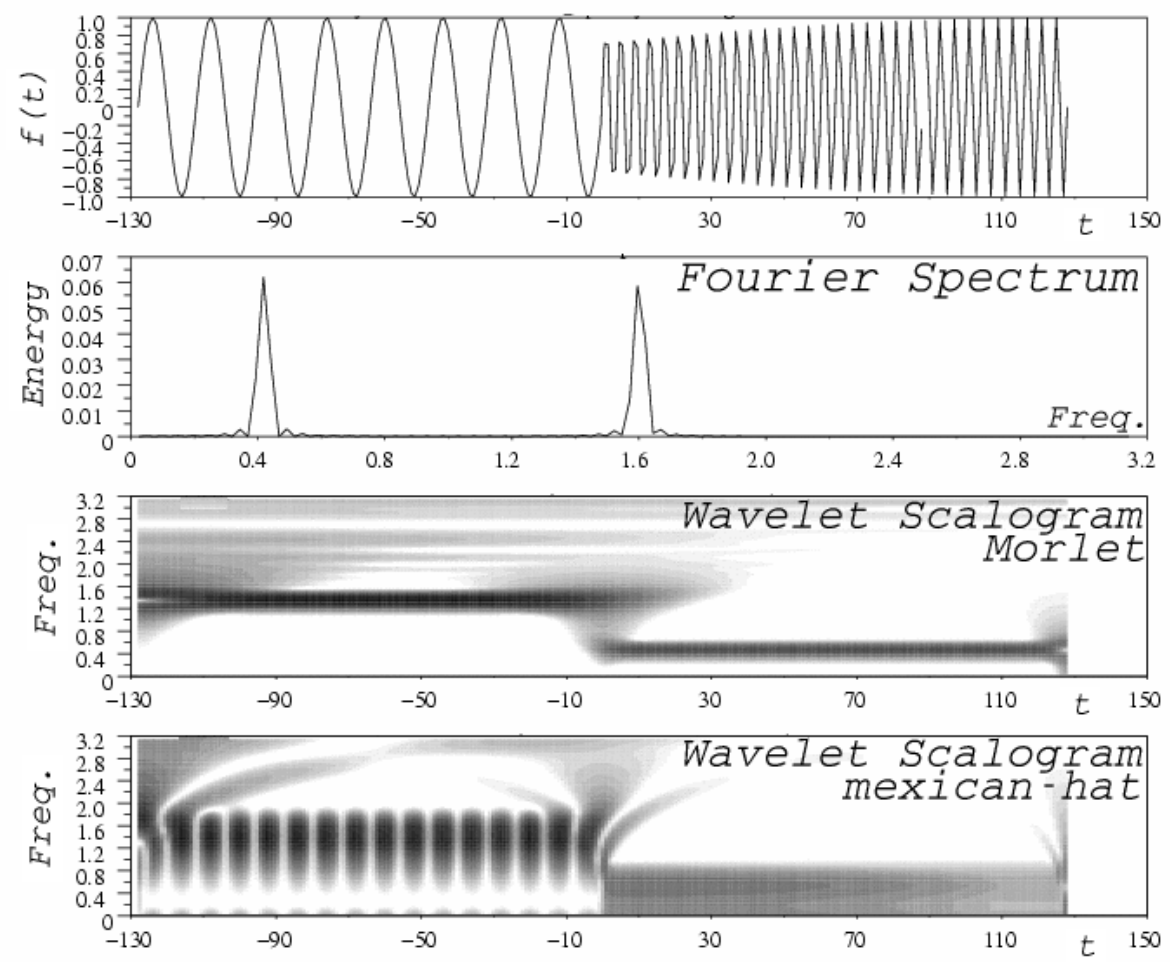

Figure 6. Representation of an oscillatory function with low and high frequencies spatially displaced and the respective Fourier spectrum and wavelet scalogram for Morlet and mexican hat mother-wavelet.

- When the analysis is focused on amplitude and phase changes, a complex wavelet, as the Morlet wavelet, can be the most appropriate. This helps to retrieve the oscillatory behavior of the data.

- In an exploratory analysis of data, non-orthogonal wavelet functions seem helpful, because they allow a redundancy in the information.

- To synthesize data and make compressions, orthogonal wavelet functions are used, since they represent the signal in a more compact way.

- When a quantitative information about a process is needed, orthogonal wavelet functions are the better choice (Kumar and Foufoula-Georgoiu, 1997). When only the wavelet spectrum is analyzed qualitatively, this choice does not seem to affect the results. This has been established by Katul et al. (1994) and Torrence and Compo (1998), for turbulence data and series of climatic data.

\section{Applications}

In the analysis, wavelet techniques are basically used in two ways: as an integration nucleus of the analysis to get information about the processes and/or as a characterization basis of the processes. Some selected papers, here shortly described, 
reveals applications in a wide range of phenomena. Ranging from issues related to atmospheric-ocean interactions to nearby space conditions, all aspects are related to the atmosphere as a whole.

Several authors have discussed the problem of choosing the Daubechies orthogonal wavelet functions for turbulent signals, for example Katul and Vidakovic (1996, 1998); Vidakovic (2000). They have found that the best choice is a function that produce less unbalance on the signal energy, i.e., in which less coefficients are needed to represent the signal. These authors have developed a threshold procedure, that they denominate as Lorentz threshold, to identify the most relevant coefficients.

Weng and Lau (1994) have studied the organization of the tropical convection in the west Pacific, using the DWT together with the Haar wavelet and the CWT together with the Morlet wavelet. Initially, they used the tool in an application with the Haar wavelet in synthetic time series of dynamical systems, with double period. Subsequently they applied this recognition pattern to an infrared data series, obtained from satellite high resolution images, using both Haar wavelet and Morlet wavelet.

Liu (1994) has defined a coherence wavelet function using a Morlet wavelet to study the interactions between the wind and the oceanic waves. Torrence and Compo (1998) have discussed the practical applicability of such information in characterizing the cross correlation, since there are some difficulties in the analysis of the resulting information. In these study, the conventional treatment given to the Fourier analysis affects the temporal localization of the wavelet analysis.

Fast numerical algorithms were developed for representations of periodic functions in bi-orthogonal multi-resolution analysis in two dimensions. It had showed that in the Fourier domain the decomposition and reconstruction algorithms had a matrix representation in terms of permutations and block diagonal matrices. Illustrative examples using bi-orthogonal spline wavelets applied to METEOSAT meteorological satellite images compression are presented in Fig. 7. In these examples the bi-orthogonal family $\left\{\phi_{1}, \phi_{1,5}\right\}$ are used. The procedure for compression is a simple truncation: we only keep the wavelet coefficients larger than a certain threshold $\epsilon$ (Domingues et al., 1995).

Briggs and Levine (1997) have applied DWT techniques in an exploratory analysis, in checking forecasting fields since the conventional measurements still reveals inadequate. This technique allowed a convenient compacting and filtering the fields partitioning, what helps in the physical interpretation of the results.

Torrence and Compo (1998) have investigated time series associated to El Niño/south oscillations phenomena to compare wavelet analysis techniques with the results already known. These authors have implemented a Monte Carlo technique to set up the confidence limits in the variance wavelet analysis in sea surface temperature time series. Periods between 2-8 years are found in the data sets before 1920 and 
(a)

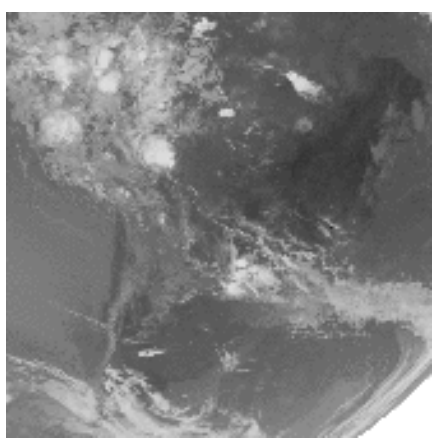

(b)

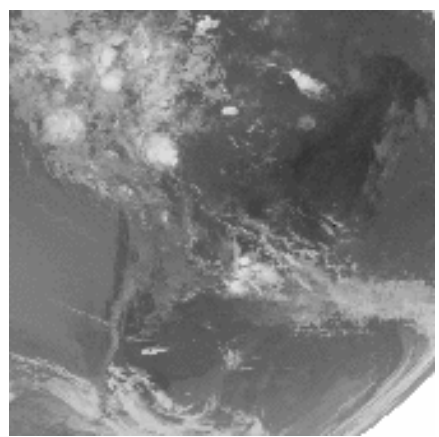

(c)

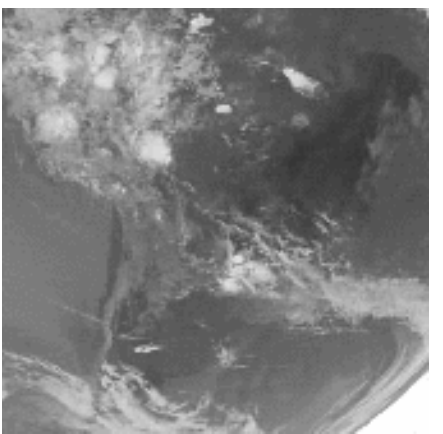

Figure 7. Illustrative example using Daubechies bi-orthogonal spline wavelet applied to METEOSAT image compression. (a) the original image, corresponding to November $21^{\text {th }}$, 1994 at 9:00am local time $\left(45^{\circ} \mathrm{W}\right)$ is shown; (b) reconstruction images using $\phi=\phi_{1}$ and $\phi^{*}=\phi_{1,5}$, and $40 \%$ of the 262,144 wavelet coefficients, those that are larger than the threshold $\epsilon=10$; and (c) the same using $\epsilon=100$ and only $8 \%$ of the wavelet coefficients. Source: Domingues et al. (1995)

2-4 years after 1960. This shows the non stationarity of the phenomena in the time scales presents in the data set.

The DWT can also be used to discriminate mesocyclones in Doppler radar data (Desrochers and Yee, 1999) and to characterize the structures of convective systems (Yano et al., 2001b,a).

In relation to the solar irradiance and climatic reconstructions, Oh et al. (2003) have conducted a multi-resolution time series analysis. The decomposition through the DWT has been made in order to facilitate the identification of the common characteristics between these time series and the climatic forcing physically associated.

The CWT together with the Morlet wavelet has been used in the detection and processing of magnetotelluric transients originated by atmospheric electrical discharges (Zhang et al., 1997). To examine the transient signals in audio-frequencies, the dominant energy sources are concentrated in thunderstorms both nearby and at great distances. The energy associated with such transients is well localized in time and therefore, the CWT can be used in the decomposition of the recorded data and in the amplitude and phase analysis of such pulses, distinguishing them from the noisy background.

More recently, in a preliminary study, Ageyev et al. (2003) have used the DWT together with the Haar wavelet to analyze sferics signals produced by lightnings, in order to obtain information on the electromagnetic field, morphological structure of the ionized channel and on the behavior of the discharge electric current.

Lawrence and Jarvis (2003), studying simultaneous observations of planetary waves from 30 to $220 \mathrm{~km}$, used the conjugate Fourier transform together with the CWT using 
Morlet. This analysis has shown that the relation between planetary waves activity at different altitudes have a high degree of complexity, since there are pulses localized at several altitudes and these series show a non-continuous behavior.

In Fig. 8, an example of DWT applied to geomagnetic minutely signals from Kakioka (Japan) using Daubechies orthogonal wavelet family 2 is presented. From top to bottom in this figure, the Dst index, the H-component of the geomagnetic field and the first three levels of the wavelet coefficients are presented for the moderate storm from November $7-8,1978$. These abrupt variations of the horizontal component of the geomagnetic field are emphasized by the largest amplitudes of the wavelet coefficients and the storm period is identified (Mendes et al., 2004).

Analyzing geomagnetic time series, Kovács et al. (2001), have used a Daubechies orthogonal DWT to identify and to isolate the intermittent sections of the signal from the background noise. Some coherent events, could also be statistically analyzed: Among other multi-scale tools. Lui (2002) used a CWT together with a Morlet wavelet to analyze the wavelike characteristics of magnetic and electric data of phenomena as auroras occurring in the magnetosphere/ionosphere coupling regions. They have defined a bi-coherence wavelet analysis to examine the coupling on non-linear wave, based in the co-spectrum wavelet.

Fligge et al. (1999) have used successfully the CWT together with the Morlet wavelet to treat more objectively the solar cycle variations correlated to some features of the earth's climate.

In a field where such techniques are not so disseminate, as cosmic rays studies, Kudela et al. (2001) have applied the CWT together with the Morlet wavelet using the same methodology proposed by Torrence and Compo (1998) to a long range time series obtained by neutron monitors. Transitions among the detected frequencies have been clearly identified in the wavelet spectra.

Solutions to many interesting atmospheric flow problems may exhibit localized singular features, such as sharp transition layers, propagating steep fronts or pronounced spikes. Reliable approximations of these problems present a challenging computational task. Uniform griding is not a practical option since high resolution is only needed in small regions, where irregularities occur. Therefore, significant improvements in accuracy and computational efficiency may be obtained by economical wavelet adapting function representation on sparse grid points to the numerical solution of partial differential equations as discussed by Domingues et al. (2003).

Although the possible applications of this technique is far from being exhausted, the works mentioned in this work, are evidences of the comprehensiveness of its use in signal analysis. 

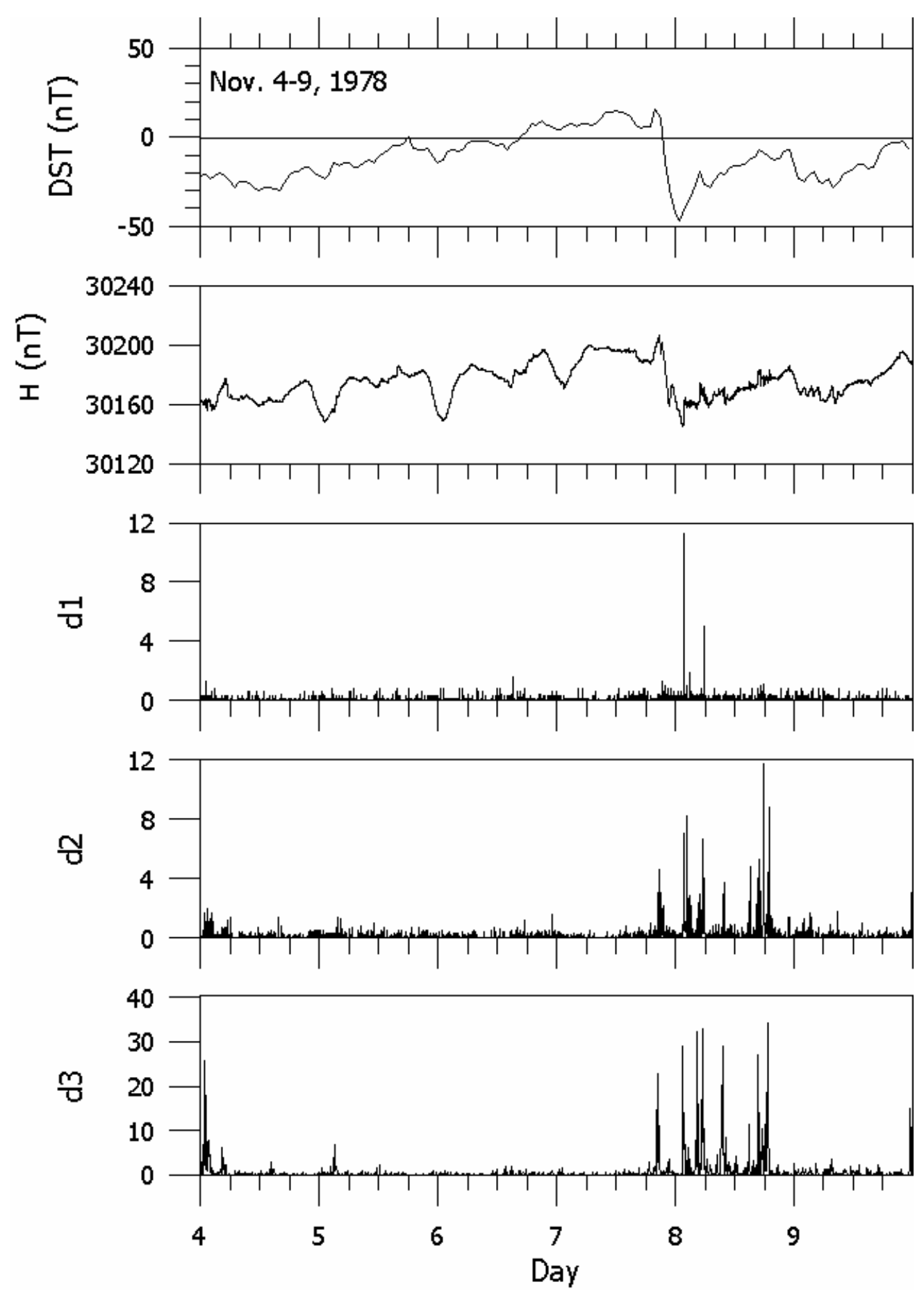

Figure 8. Geomagnetic field data set for Kakioka station and wavelet coefficients amplitudes $d 1,2,3=\left|d^{1,2,3}\right|$, respectatively at levels 1,2 and 3 , for the geomagnetic storm of November, 7-8, 1978. 


\section{Final remarks}

The wavelet transform reveals where the energy is transferred in the analyzed signals and at which frequencies this occurs. From the physical point of view this evidenced in a certain way, the real meaning of this tool. This work is far from being a complete review on the subject, but it is an attempt to characterize the efforts oriented to the atmospheric wavelet applications, by a selection of some relevant updated published papers.

To assure a complete understanding and to check the consistency of the obtained results in the investigation of atmospheric phenomena using this technique, a good knowledge of the wavelet tool is required.

There are several wavelet families, as the Meyer wavelet that has been used by Yano et al. (2001b,a), the wavelet packets defined in Coifman et al. (1992); Meyer (1989) and the multi-wavelet worked out by Lilly and Park (1995); Zanandrea et al. (2000). In the great majority of atmospheric applications, the Morlet, mexican hat, Haar and Daubechies families of wavelet functions have been extensively used. This preference may be attributed to the availability of special softwares to derive CWT and DWT based on these wavelet families.

In the Atmospheric Sciences, the applications of wavelet analysis are systematically increasing, as it can be noticed by the number of publications in the relevant journals and congresses in the last decade. In spite of the initial effort required for the proper formalism, currently the wavelet transforms had turn out to be a very useful tool in atmospheric signal analysis, creating an encouraging new horizon to the research activities.

\section{Appendix}

Some relevant web pages in wavelet analysis: 
Theoretical information

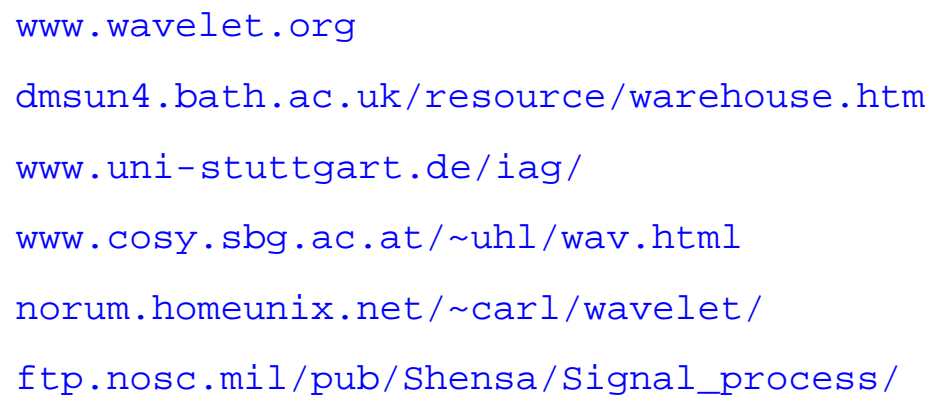

$\underline{\text { Softwares }}$

$\begin{array}{ll}\text { Amara } & \text { www.amara.com/current/wavesoft.html } \\ \text { FracLab/Scilab } & \text { www-rocq.inria.fr/scilab/contributions.html } \\ \text { Lifting } & \text { www.cs.kuleuven.ac.be/ wavelets/ } \\ \text { Morlet } & \text { ftp.nosc.mil/pub/Shensa/Signal_process/ } \\ \text { Numerical Recipes } & \text { www.nr.com/public-domain.html } \\ \text { Rice } & \text { www-dsp.rice.edu/software/rwt.shtml } \\ \text { Wavelab/MatLab } & \text { www-stat.stanford.edu/ wavelab/ } \\ \text { WPLab } & \text { www.math.wustl.edu/ victor/software/wPLab/ } \\ \text { WaveTresh/R } & \text { www.stats.bris.ac.uk/ wavethresh/software } \\ \text { Torrence and Compo (1998) } & \text { paos.colorado.edu/research/wavelets/software.html }\end{array}$

\section{Acknowledgements}

The authors wish to thank CNPq through grant PCI-INPE 382465/01-6.

\section{References}

Ageyev, A., Gandelli, A., Zich, R. E., 2003. Analysis of lightning signals by a haarbased wavelet approach. In: 5th EMC International Symposium.

Briggs, W. M., Levine, R. A., Jun. 1997. Wavelets and field forecast verification. Monthly Weather Review 125 (6), 1329-1341.

Chui, C., 1992a. An Introdution to Wavelets. Vol. 1. Academic Press.

Chui, C. (Ed.), 1992b. Wavelets:A tutorial in theory and applications. Vol. 2. Academic Press.

Chui, C. (Ed.), 1994. Wavelets theory, algorithms. Academic Press. 
Coifman, R. R., Meyer, Y., Wickerhauser, M. V., 1992. Wavelets and their applications. Jone and Bartlett, Ch. Wavelet analysis and signal processing.

Daubechies, I., 1990. The wavelet transform, time-frequency localization and signal analysis. IEEE Transactions on information theory 5, 961-1005.

Daubechies, I., 1992. Ten Lectures on Wavelets. Vol. 61 of CBMS-NSF Regional Conference (Series in Applied Mathematics). SIAM, Philadelphia, PA.

Daubechies, I., Mallat, S., Willsky, A., 1992. Introduction to the special issue on wavelet trasnforms and multiresolution signal analysis. IEEE Trans. Inf. Theory 38 (2), 528-531.

Desrochers, P. R., Yee, S. Y. K., 1999. Wavelet applications for mesocyclone identification in doppler radar observations. Journal of Applied Meteorology 38 (7), 965-980.

Domingues, M., Gomes, S. M., Diaz, L. M. A., 2003. Adaptive wavelet representation and differentiation on block-structured grids. journal applied numerical mathematics. Journal Applied Numerical Mathematics 47 (3-4), special issue: Applied and Computational Mathematics: Selected Papers of the Fourth PanAmerican Workshop.

Domingues, M. O., Gomes, S., Cortina, E., Apr. 17-21, 1995. Biorthogonal wavelets applied to meteosat image compressing. In: Szu, H. H. (Ed.), Wavelet and Aplications II. Vol. 2491. Spie, Orlando, FL, pp. 726-733.

Domingues, M. O., Prasad, G. S., Sá, L. D. A., von Randow, C., Manzi, A. O., Kruijt, B., 2002. Evidence of non-existence of a 'spectral-gap' in turbulent data measured above rondonia, brazil. Part I: Amazonian Forest. In: Second LBA scientific conference. LBA, (abstract) <http://lba.cptec.inpe.br/lba/>.

Farge, M., 1992. wavelet transform and their applications to turbulence. Annual Review of Fluid Mechanics 24, 395-457.

Flandrin, P., 1988. Time-frequency and time-scale. In: 4th Acustic, Speech and signal processing workshop on spectrum estimation modeling. New York, pp. 77-80.

Fligge, M., Solank, S. K., Beer, J., 1999. Determination of solar cycle length variations using the continuous wavelet transform. Astronomy and Astrophysics 346, 313-321.

Foufola-Georgoiu, E., Kumar, P. (Eds.), 1994. Wavelets in Geophysics. Vol. 4 of Wavelet Analisys and its Applications. Academic Press.

Gabor, D., 1946. Theory of communication. Journal of I.E.E. 93, 429-441.

Gambis, D., 1992. Wavelet transform analysis of the length of the day and the elniño/southern oscilation variation at intraseasonal and interannual time scales. Annual Geophysicae 10, 429-437.

Gao, W., Li, B. L., 1993. Wavelet analysis of coherent strunts at the atmosphereforest interface. Journal of Applied Meteorology 32, 1717-1725.

Houdré, C., 1994. Wavelets mathematics and applications. Studies in Advanced Mathematics. CRC Press, Boca Raton, Ch. Wavelet, probability, and applications: some bridges, pp. 365-398.

Jawerth, B., Sweldens, W., 1994. An overview of wavelet based multiresolution analyses. SIAM Review 36 (3), 377-412. 
Katul, G., Vidakovic, B., 1996. The partitioning of attached and detached eddy motion in the atmospheric surface layer using lorentz wavelet filtering. Boundary layer Meteorology 77 (2), 153-172.

Katul, G., Vidakovic, B., 1998. Identification of low-dimensional energy containing/flux transporting eddy motion in atmospheric surface layer using wavelet thresholding methods. Journal of the atmospheric sciences 55 (3), 377-389.

Katul, G. G., Albertson, J. D., Chu, C. R., Parlange, M. B., 1994. Wavelets in Geophysics. Vol. 4 of Wavelet Analysis and its Aplications. Academic Press, San Diego, Ch. Intermittency in atmospheric surface layer turbulence: the orthonormal wavelet representation, pp. 81-105.

Kovács, P., Carbone, V., Vörös, Z., 2001. Wavelet-based filtering of intermitents events from geomagnetic time-series. Planetary and Space Science 49, 12191231.

Kudela, K., Storini, M., Antalová, A., Rybák, J., 2001. On the wavelet approach to cosmic ray variability. In: ICRC. Copernicus Gesellschaft.

Kumar, P., Foufoula-Georgoiu, E., 1997. Wavelet analisys for geophysical aplications. Reviews of Geophysics 35 (4), 385-412.

Lau, K.-M., Weng, H., 1995. Climate signal detection using wavelet transform: How to make a time series sing. Bulletin of the Americam Meteorological Society 76 (12), 2391-2402.

Lawrence, A. R., Jarvis, M. J., 2003. Simultaneous observations of planetary waves from 30 to 220km. Journal of Atmospheric and Solar-Terrestrial Physics 65, 765-777.

Lilly, J. M., Park, J., 1995. Multwavelet spectral and polarization analyses of seismic records. Geophys. J. Int. 122, 1001-1021.

Liu, P. C., 1994. Wavelets in Geophysics. Vol. 4 of Wavelet Analysis and its Aplications. Academic Press, San Diego, Ch. Wavelet spectrum analysis and ocean wind waves, pp. 151-166.

Lui, A. T. Y., 2002. Multiscale phenomena in the near-earth magnetosphere. Journal of Atmospheric and Solar-Terrestrial Physics 64, 125-143.

Mallat, S., 1991. Multiresolution approximations and wavelets orthonormal bases. Trans. of American Mathematical Society 315, 334-351.

Mendes, O. J., Domingues, M. O., Mendes da Costa, A., Mar 2004. Wavelet analysis applied to magnetograms. In: VII COLAGE Abstracts. ALAGE, ALAGE, Atibaia, São Paulo, p. 177, <http://www.cea.inpe.br/colage>.

Meneveau, C., 1991. Analisys of turbulence in the orthonormal wavelet representation. Journal of Fluid Mechanics 232, 469-520.

Meyer, Y., 1989. Wavelets. Springer Verlag, Berlin.

Meyer, Y., 1990. Ondelettes et Operateurs. Hermann, Paris.

Morlet, J., 1983. Sampling Teory and wave propagation. Springer.

Oh, H.-S., Ammann, C. M., Naveau, P., Nychka, D., Otto-Bliesner, B. O., 2003. Multi-resolution time series analysis applied to solar irradiance and climate reconstruction. Journal of Atmospheric and Solar-Terrestrial Physics 65, 191-201.

Percival, D., Walden, A., 2000. Wavelets Methods for Time Series Analysis. Cambridge University. 
Strang, G., Nguyen, T., 1996. Wavelet and filters bank. Wellesley-Cambridge, , http://saigon.ece.wisc.edu/ waveweb/tutorials/book.html.

Sweldens, W., Schröder, P., 1995. Building your own wavelet at home. In: Computer Graphics Proceedings. ACM Siggraph, SIGGRAPH 95, (http://cm.belllabs.com/cm/ms /who/wim/papers/papers.html).

Torrence, C., Compo, G. P., 1998. A practical guide to wavelet analysis. Bulletin of the American Meteorological Society.

Vidakovic, B., 2000. Statistical Modeling by Wavelets. Probability and Statistics. Wiley.

Weng, H.-Y., Lau, K.-M., 1994. Wavelet, period-doubling and time frequency localization with application organization of convection over the tropical western Pacific. Journal of Atmospheric Science 51 (17), 2523-2541.

Yano, J.-I., Moncrieff, M. W., Wu, X., 2001a. Wavelet analysis of simulated tropical convective cloud systems. part ii: Decomposition of convective-scale and mesoscale structure. Journal of the Atmospheric Sciences 58 (8), 868-876.

Yano, J.-I., Moncrieff, M. W., Wu, X., Yamada, M., 2001b. Wavelet analysis of simulated tropical convective cloud systems. part i: Basic analysis. Journal of the Atmospheric Sciences 58 (8), 850-867.

Zanandrea, A., Rodrigues Neto, C., Rosa, R. R., Ramos, F. M., 2000. Analysis of geomagnetic pulsations using multiwavelets spectral and polarization method. Physica A.

Zhang, Y., Goldak, D., Paulson, K., May 1997. Detection and processing of lightning-sourced magnetotelluric transients with the wavelet transform. IEICE Transaction Fundamentals E80A (5), 849-858.

\section{List of Tables}

$1 \quad$ Scale coefficient $h(k)$ for Daubechies orthogonal functions

\section{List of Figures}

1 Time/space vs frequency plane representation. Proportional variations of time and frequency intervals are presented on the left. On the right, the dilatation/contraction of a wavelet function in those respective intervals is shown.

2 Graph of real and complex part of Morlet mother-wavelet

3 Graph of mexican-hat mother-wavelet. 
4 Typical examples of Daubechies orthogonal scale and wavelet function families.

5 One example of wavelet spectrum of the zonal wind component and co-spectrum of temperature and vertical wind component of turbulent signals from a micro-meteorological tower in Rebio Jaru (Amazon region) in (a) and (b) respectively. In (b), a standard power law function is also shown in the upper part.

6 Representation of an oscillatory function with low and high frequencies spatially displaced and the respective Fourier spectrum and wavelet scalogram for Morlet and mexican hat mother-wavelet.

7 Illustrative example using Daubechies bi-orthogonal spline wavelet applied to METEOSAT image compression. (a) the original image, corresponding to November $21^{\text {th }}, 1994$ at 9:00am local time $\left(45^{\circ} \mathrm{W}\right)$ is shown; (b) reconstruction images using $\phi=\phi_{1}$ and $\phi^{*}=\phi_{1,5}$, and $40 \%$ of the 262,144 wavelet coefficients, those that are larger than the threshold $\epsilon=10$; and (c) the same using $\epsilon=100$ and only $8 \%$ of the wavelet coefficients.

8 Geomagnetic field data set for Kakioka station and wavelet coefficients amplitudes $d 1,2,3=\left|d^{1,2,3}\right|$, respectatively at levels 1, 2 and 3, for the geomagnetic storm of November, 7-8, 1978. 